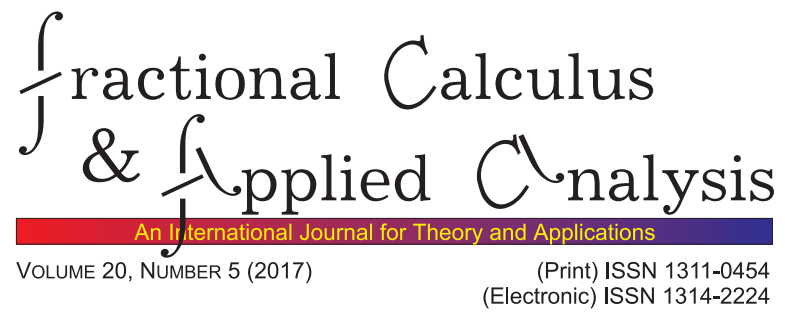

RESEARCH PAPER

\title{
WELL-POSEDNESS AND NUMERICAL APPROXIMATION OF TEMPERED FRACTIONAL TERMINAL VALUE PROBLEMS
}

\author{
Maria Luísa Morgado ${ }^{1}$, Magda Rebelo ${ }^{2}$ \\ Dedicated to Professor Virginia Kiryakova on the occasion \\ of her 65th birthday and the 20th anniversary of FCAA
}

\begin{abstract}
For a class of tempered fractional terminal value problems of the Caputo type, we study the existence and uniqueness of the solution, analyze the continuous dependence on the given data, and using a shooting method we present and discuss three numerical schemes for the numerical approximation of such problems. Some numerical examples are considered in order to illustrate the theoretical results and evidence the efficiency of the numerical methods.
\end{abstract}

MSC 2010: Primary 65L05; Secondary 34A08, 26Axx

Key Words and Phrases: tempered fractional derivatives, Caputo Derivative, terminal value problem, numerical methods, shooting method

\section{Introduction}

In this work we analyze a class of tempered terminal value problems for tempered fractional ordinary differential equations of order $\alpha$, with $0<\alpha<1$ :

$$
\begin{aligned}
& { }_{0} \mathbb{D}_{t}^{\alpha, \lambda}(y(t))=f(t, y(t)), \quad t \in[0, a], \\
& e^{\lambda a} y(a)=y_{a},
\end{aligned}
$$

(c) 2017 Diogenes Co., Sofia

pp. 1239-1262, DOI: $10.1515 /$ fca-2017-0065

DE GRUYTER 
where $f$ is a suitably behaved function and $\mathbb{D}_{0}^{\alpha, \lambda}(y(t))$ denotes the left-sided Caputo tempered fractional derivative of order $\alpha>0$, where the tempered parameter $\lambda$ is nonnegative.

The left-sided Caputo tempered fractional derivative can be given trough the definition of the Caputo derivative (see [7] for example). In the particular case where $0<\alpha<1$ it reads:

$$
\begin{aligned}
{ }_{0} \mathbb{D}_{t}^{\alpha, \lambda}(y(t)) & =e^{-\lambda t}{ }_{0} \mathcal{D}_{t}^{C, \alpha}\left(e^{\lambda t} y(t)\right) \\
& =\frac{e^{-\lambda t}}{\Gamma(1-\alpha)} \int_{0}^{t} \frac{1}{(t-s)^{\alpha}} \frac{d\left(e^{\lambda s} y(s)\right)}{d s} d s,
\end{aligned}
$$

where ${ }_{0} \mathcal{D}_{t}^{C, \alpha}$ denotes the Caputo fractional derivative (see [3]).

Note that if $\lambda=0$ then the Caputo tempered fractional derivative reduces to the Caputo fractional derivative, and therefore, Caputo derivatives can be regarded as a particular case of Caputo tempered derivatives.

Fractional differential equations of Caputo-type have been investigated extensively in the last decades and many significant contributions were provided by researchers of several areas as mathematics, physics and engineering, making Fractional Calculus as one of the most hot and current research topics. Recently, some attention has been devoted to tempered fractional differential equations, because the later ones revealed to model more realistically some phenomena (see [10, [12, [8] and the references therein for details). Even so, the literature is not so vast for this type of equations, as it is for fractional differential equations in the Caputo sense. As it happens with non-tempered fractional differential equations, the analytical solution is usually impossible to obtain and in the cases where it can be determined, its representation in terms of a series makes it difficult to handle. Therefore, the development of numerical methods for this type of fractional differential equations is also crucial. With this respect, some approaches have already been reported. In [1], the authors propose a finite difference formula for tempered fractional derivatives and introduce a temporal and spatial second-order Crank-Nicolson method for the spacefractional diffusion equation. In [6] and [7] a Jacobi-predictor-corrector algorithm is presented for tempered ordinary initial value problems. The authors in [9] present a finite difference scheme to solve fractional partial differential models in finance. In [13], spectral methods are derived for the tempered advection and diffusion problems.

To the best of our knowledge, tempered terminal value problems have never been investigated. Therefore, after analyzing the well-posedness of such problems, we consider a simple approach for the numerical approximation of the solution, which is based on the relationship between tempered and non-tempered Caputo derivatives. 
WELL-POSEDNESS AND NUMERICAL APPROXIMATION ... 1241

The paper is organized in the following way: in the next Section 2 we establish sufficient conditions for the existence and uniqueness of the solution of problems of the type (1.1)-(1.2). Then we investigate the continuous dependence of the solution on the given data. Section 4 is devoted to the derivation of numerical schemes and finally in Section 5 we present and discuss several numerical examples. The paper ends with some conclusions and plans for further investigation.

\section{Existence and uniqueness of the solution}

From [7] we have the following two results for initial value problems.

Lemma 2.1. ([7]) If the function $f(t, u)$ is continuous, then the initial value problem

$$
\left\{\begin{array}{l}
{ }_{0} \mathbb{D}_{t}^{\alpha, \lambda}(y(t))=f(t, y(t)), \quad t \in[0, a], \\
{\left.\left[\frac{d^{k}}{d t^{k}}\left(e^{-\lambda t} y(t)\right)\right]\right|_{t=0}=c_{k}, \quad k=0,1, \ldots, n-1,}
\end{array}\right.
$$

is equivalent to the nonlinear Volterra integral equation of the second kind

$$
y(t)=\sum_{k=0}^{n-1} c_{k} \frac{e^{-\lambda t} t^{k}}{\Gamma(k+1)}+\frac{1}{\Gamma(\alpha)} \int_{0}^{t} e^{-\lambda(t-s)}(t-s)^{\alpha-1} f(s, y(s)) d s,
$$

where $n-1<\alpha<n$ and $\alpha \geq 0$.

In the particular case where $0<\alpha<1$, we have

$$
y(t)=c_{0} e^{-\lambda t}+\frac{1}{\Gamma(\alpha)} \int_{0}^{t} e^{-\lambda(t-s)}(t-s)^{\alpha-1} f(s, y(s)) d s .
$$

Theorem 2.1. ([7]) Let $n-1<\alpha<n, n \in \mathbb{N}^{+}, \lambda \geq 0$ and $a \in \mathbb{R}$ such that $a>0$. Furthermore, let the function $f(t, y)$ be a continuous function such that $f(t, y) \in L([0, a])$, for any $y \in B$, where $B$ is an open set in $\mathbb{R}$, and $f(t, y)$ also satisfies a Lipschitz condition with respect to the second variable, then the fractional differential equation (2.1) has a unique solution $u(t) \in C^{n}([0, a])$.

Next, we extend these results to terminal boundary problems. In what follows, the Caputo tempered fractional derivative of order $\alpha$ will be simply denoted by $\mathbb{D}^{\alpha, \lambda}(y(t))$.

The Caputo tempered fractional derivative of order $\alpha$, with $\alpha \in(0,1)$ satisfies

$$
\begin{aligned}
& \mathbb{I}^{\alpha, \lambda}\left(\mathbb{D}^{\alpha, \lambda}(u(t))\right)=u(t)-e^{\lambda t} u(0), \\
& \mathbb{D}^{\alpha, \lambda}\left(\mathbb{I}^{\alpha, \lambda}(u(t))\right)=u(t)
\end{aligned}
$$


where $\mathbb{I}^{\alpha, \lambda}$ is the Riemann-Liouville tempered fractional integral given by

$$
\mathbb{I}^{\alpha, \lambda}(u(t))=e^{-\lambda t} \mathbb{I}^{\alpha}\left(e^{\lambda t} u(t)\right)=\frac{1}{\Gamma(\alpha)} \int_{0}^{t} e^{-\lambda(t-s)}(t-s)^{\alpha-1} u(s) d s,
$$

and $\mathbb{I}^{\alpha}$ denotes the Riemann-Liouville fractional integral.

If $y$ satisfies the fractional differential equation (1.1), then applying the Riemann-Liouville fractional integral at the both sides of equation and taking property (2.3) into account, we conclude that the solution $y$ satisfies the following integral equation

$$
\begin{aligned}
e^{\lambda t} y(t) & =y(0)+\frac{1}{\Gamma(\alpha)} \int_{0}^{t} e^{\lambda s}(t-s)^{\alpha-1} f(s, y(s)) d s \\
& =y(0)+\frac{1}{\Gamma(\alpha)} \int_{0}^{a} e^{\lambda s}(a-s)^{\alpha-1} f(s, y(s)) d s \\
& -\frac{1}{\Gamma(\alpha)}\left(\int_{0}^{a} e^{\lambda s}(a-s)^{\alpha-1} f(s, y(s)) d s\right. \\
& \left.+\int_{0}^{t} e^{\lambda s}(t-s)^{\alpha-1} f(s, y(s)) d s\right) \\
& =y_{a}-\frac{1}{\Gamma(\alpha)} \int_{0}^{a} e^{\lambda s}(a-s)^{\alpha-1} f(s, y(s)) d s \\
& +\frac{1}{\Gamma(\alpha)} \int_{0}^{t} e^{\lambda s}(t-s)^{\alpha-1} f(s, y(s)) d s
\end{aligned}
$$

Therefore, if $y$ is a solution of the fractional boundary value problem (FBVP) (1.1)-(1.2) then $y$ is a solution of the integral equation

$$
\begin{aligned}
y(t) & =y_{a} e^{-\lambda t}-\frac{e^{-\lambda t}}{\Gamma(\alpha)} \int_{0}^{a} e^{\lambda s}(a-s)^{\alpha-1} f(s, y(s)) d s \\
& +\frac{e^{-\lambda t}}{\Gamma(\alpha)} \int_{0}^{t} e^{\lambda s}(t-s)^{\alpha-1} f(s, y(s)) d s .
\end{aligned}
$$

Next, we establish sufficient conditions for the existence and uniqueness of solutions of the FBVP (1.1)-(1.2). The proof will be based on the Banach's fixed point theorem. We just establish the existence and uniqueness of the solution on the interval $[0, a]$, since the existence and uniqueness for $t>a$ is inherited from the corresponding initial value problem theory (see [7] for details).

Define the set $\Omega_{\gamma}=\left\{y \in C([0, a]):\left\|y-y_{a} e^{-\lambda t}\right\|_{[0, a]} \leq \gamma\right\}$, where the norm $\|\cdot\|_{[0, a]}$ is defined by $\|\cdot\|_{[0, a]}=\max _{t \in[0, a]}|g(t)|$, for all $g \in C([0, a])$ and

$$
\gamma=\frac{2 a^{\alpha}\|f\|_{[0, a]} e^{\lambda a}}{\Gamma(1+\alpha)}
$$


The set $\Omega_{\gamma}$ is a closed subset of the Banach space of all continuous functions on $[0, a]$, equipped with the norm \|\|$_{[0, a]}$, and since the function $y(t)=$ $y_{a} e^{-\lambda t} \in \Omega_{\gamma}$, it is nonempty. On $\Omega_{\gamma}$, let us define the operator

$$
\begin{aligned}
(\mathcal{A} y)(t) & =y_{a} e^{-\lambda t}-\frac{1}{\Gamma(\alpha)} \int_{0}^{a} e^{-\lambda(t-s)}(a-s)^{\alpha-1} f(s, y(s)) d s \\
& +\frac{1}{\Gamma(\alpha)} \int_{0}^{t} e^{-\lambda(t-s)}(t-s)^{\alpha-1} f(s, y(s)) d s .
\end{aligned}
$$

Using this operator, the integral equation can be rewritten as $y=\mathcal{A} y$, and if the operator $\mathcal{A}$ has a unique fixed point on $\Omega_{\gamma}$ then the FBVP (1.1)-(1.2) has a unique continuous solution. Using the Banach's fixed point theorem, under some assumptions on $f$, we prove the existence and uniqueness result on the next theorem.

TheOREM 2.2. Let $D=[0, a] \times\left[y_{a} e^{-\lambda a}-\gamma, y_{a} e^{-\lambda a}+\gamma\right]$, with $\gamma$ given by (2.8), and assume that the function $f: D \rightarrow \mathbb{R}$ is continuous for all $t \in[0, a]$. We further assume that the function $f$ fulfills a Lipschitz condition with respect to the second variable, meaning that there exists $L>0$ such that it holds

$$
|f(t, y)-f(t, z)| \leq L|y-z|, \quad \text { for } \quad y, z \in \Omega_{\gamma} .
$$

If the Lipschitz constant $L$ is such that $L<\frac{\Gamma(\alpha+1)}{2 a^{\alpha} e^{\lambda a}}$, then $\mathcal{A}$ maps $\Omega_{\gamma}$ into itself and it is a contraction:

$$
\|\mathcal{A} y-\mathcal{A} z\|_{[0, a]} \leq\|y-z\|_{[0, a]} \quad \text { for } \quad y, z \in \Omega_{\gamma} .
$$

Hence equation (2.7) has a unique solution $y^{*} \in \Omega_{\gamma}$, which is the unique fixed point of $\mathcal{A}$.

P r o o f. Let $y \in \Omega_{\gamma}$. First, we show that $\mathcal{A} y \in \Omega_{\gamma}$.

From the definition of $\mathcal{A}$ we have

$$
\begin{aligned}
\left|\mathcal{A} y-y_{a} e^{-\lambda t}\right| & =\frac{e^{-\lambda t}}{\Gamma(\alpha)} \mid \int_{0}^{a} e^{\lambda s}(a-s)^{\alpha-1} f(s, y(s)) d s \\
& +\int_{0}^{t} e^{\lambda s}(t-s)^{\alpha-1} f(s, y(s)) d s \mid \leq \frac{\|f\|_{[0, a]} e^{\lambda a}}{\alpha \Gamma(\alpha)}\left(a^{\alpha}+t^{\alpha}\right) .
\end{aligned}
$$

Then

$$
\left\|\mathcal{A} y-y_{a} e^{-\lambda t}\right\|_{[0, a]} \leq \frac{2 a^{\alpha}\|f\|_{[0, a]} e^{\lambda a}}{\Gamma(\alpha+1)}=\gamma,
$$

which implies that $(\mathcal{A} y) \in \Omega_{\gamma}$. 
Now we prove that $\mathcal{A}$ is a contraction on $\Omega_{\gamma}$, with $\gamma$ defined by (2.8). For $y, z \in \Omega_{\gamma}, t \in[0, a]$, we have

$$
\begin{aligned}
& |(\mathcal{A} y)(t)-(\mathcal{A} z)(t)| \\
& \leq \frac{1}{\Gamma(\alpha)}\left(\int_{0}^{a} e^{-\lambda(t-s)}(a-s)^{\alpha-1}|f(s, y(s))-f(s, z(s))| d s\right. \\
& \left.+\int_{0}^{t} e^{-\lambda(t-s)}(t-s)^{\alpha-1}|f(s, y(s))-f(s, z(s))| d s\right) \\
& \leq \frac{2 L a^{\alpha} e^{\lambda a}}{\alpha \Gamma(\alpha)}\|y-z\|_{[0, a]}=\frac{2 L a^{\alpha} e^{\lambda a}}{\Gamma(\alpha+1)}\|y-z\|_{[0, a]}<\|y-z\|_{[0, a]} .
\end{aligned}
$$

Then, the operator $\mathcal{A}$ is a contraction on $\Omega_{\gamma}$. Finally, by the Banach fixed point principle the proof of the theorem is complete.

If the assumptions of Theorem 2.2 are satisfied, then the FBVP (1.1)(1.2) has an unique continuous solution, $y(t)$, on the interval $[0, a]$ and, in particular, a unique value for $y(0)$ exists. Therefore, there is an exact correspondence between tempered fractional boundary value problems and tempered fractional initial value problems.

\section{Continuous dependence of the solution on the data}

In order to analyze the continuous dependence of the solution on the given data we assume that problem

$$
\begin{aligned}
\mathbb{D}_{t}^{\alpha, \lambda}(y(t)) & =f(t, y(t)), \quad t \in[0, a], \\
e^{\lambda a} y(a) & =y_{a},
\end{aligned}
$$

which is equivalent to

$$
\begin{aligned}
y(t) & =y_{a} e^{-\lambda t}-\frac{e^{-\lambda t}}{\Gamma(\alpha)} \int_{0}^{a} e^{\lambda s}(a-s)^{\alpha-1} f(s, y(s)) d s \\
& +\frac{e^{-\lambda t}}{\Gamma(\alpha)} \int_{0}^{t} e^{\lambda s}(t-s)^{\alpha-1} f(s, y(s)) d s
\end{aligned}
$$

may suffer perturbations on the parameters $y_{a}, \alpha, \lambda$ and on the right-hand side function $f$, and therefore we will consider the following perturbed problems:

$$
\begin{aligned}
\mathbb{D}_{t}^{\alpha, \lambda}(z(t)) & =f(t, z(t)), \quad t \in[0, a], \\
e^{\lambda a} z(a) & =z_{a}, \\
\mathbb{D}_{t}^{\alpha-\delta, \lambda}(z(t)) & =f(t, z(t)), \quad t \in[0, a], \\
e^{\lambda a} z(a) & =y_{a},
\end{aligned}
$$


WELL-POSEDNESS AND NUMERICAL APPROXIMATION . . 1245

$$
\begin{aligned}
\mathbb{D}_{t}^{\alpha, \lambda-\delta}(z(t)) & =f(t, z(t)), \quad t \in[0, a] \\
e^{(\lambda-\delta) a} z(a) & =y_{a}, \\
\mathbb{D}_{t}^{\alpha, \lambda}(z(t)) & =\tilde{f}(t, z(t)), \quad t \in[0, a], \\
e^{\lambda a} z(a) & =y_{a},
\end{aligned}
$$

$\delta>0$, which are equivalent to the following integral equations:

$$
\begin{aligned}
z(t)= & z_{a} e^{-\lambda t}-\frac{e^{-\lambda t}}{\Gamma(\alpha)} \int_{0}^{a} e^{\lambda s}(a-s)^{\alpha-1} f(s, z(s)) d s \\
& +\frac{e^{-\lambda t}}{\Gamma(\alpha)} \int_{0}^{t} e^{\lambda s}(t-s)^{\alpha-1} f(s, z(s)) d s \\
z(t)= & y_{a} e^{-\lambda t}-\frac{e^{-\lambda t}}{\Gamma(\alpha-\delta)} \int_{0}^{a} e^{\lambda s}(a-s)^{\alpha-\delta-1} f(s, z(s)) d s \\
& +\frac{e^{-\lambda t}}{\Gamma(\alpha-\delta)} \int_{0}^{t} e^{\lambda s}(t-s)^{\alpha-\delta-1} f(s, z(s)) d s \\
z(t)= & y_{a} e^{-(\lambda-\delta) t}-\frac{e^{-(\lambda-\delta) t}}{\Gamma(\alpha)} \int_{0}^{a} e^{(\lambda-\delta) s}(a-s)^{\alpha-1} f(s, z(s)) d s \\
& +\frac{e^{-(\lambda-\delta) t}}{\Gamma(\alpha)} \int_{0}^{t} e^{(\lambda-\delta) s}(t-s)^{\alpha-1} f(s, z(s)) d s \\
z(t)= & y_{a} e^{-\lambda t}-\frac{e^{-\lambda t}}{\Gamma(\alpha)} \int_{0}^{a} e^{\lambda s}(a-s)^{\alpha-1} \tilde{f}(s, z(s)) d s \\
& +\frac{e^{-\lambda t}}{\Gamma(\alpha)}-\int_{0}^{t} e^{\lambda s}(t-s)^{\alpha-1} \tilde{f}(s, z(s)) d s
\end{aligned}
$$

respectively. In the following results the function $f$ satisfies the assumptions of Theorem 2.2 .

TheOREM 3.1. Let $y$ and $z$ be the unique solutions of problems (1.1)(1.2) and (3.2)-(3.3), respectively. Then

$$
\|y-z\| \leq \frac{1}{\beta}\left|y_{a}-z_{a}\right|
$$

where $\beta=1-\frac{2 L e^{\lambda a} a^{\alpha}}{\Gamma(\alpha+1)}$ 
P r o o f. Taking (3.1) and (3.10) into account, for any $t \in[0, a]$, we have

$$
\begin{aligned}
|y(t)-z(t)| \leq & e^{-\lambda t}\left(y_{a}-z_{a}\right) \\
+ & \frac{e^{-\lambda t}}{\Gamma(\alpha)}\left|-\int_{0}^{a} e^{\lambda s}(a-s)^{\alpha-1}(f(s, y(s))-f(s, z(s))) d s\right| \\
+ & \frac{e^{-\lambda t}}{\Gamma(\alpha)} \int_{0}^{t} e^{\lambda s}(t-s)^{\alpha-1}|f(s, y(s))-f(s, z(s))| d s \\
\leq & \left|y_{a}-z_{a}\right|+\frac{L e^{\lambda a}}{\Gamma(\alpha)} \int_{0}^{a}(a-s)^{\alpha-1}|y(s)-z(s)| d s \\
& +\frac{L e^{\lambda a}}{\Gamma(\alpha)} \int_{0}^{t}(t-s)^{\alpha-1}|y(s)-z(s)| d s .
\end{aligned}
$$

Hence

$$
\begin{aligned}
\|y-z\| \quad & \leq\left|y_{a}-z_{a}\right|+\frac{L e^{\lambda a}}{\Gamma(\alpha)}\|y-z\|\left(\int_{0}^{a}(a-s)^{\alpha-1} d s+\int_{0}^{t}(t-s)^{\alpha-1} d s\right) \\
& =\left|y_{a}-z_{a}\right|+\frac{L e^{\lambda a}}{\Gamma(\alpha)}\|y-z\|\left(\frac{a^{\alpha}}{\alpha}+\frac{t^{\alpha}}{\alpha}\right) \\
& \leq\left|y_{a}-z_{a}\right|+\frac{2 L e^{\lambda a} a^{\alpha}}{\Gamma(\alpha+1)}\|y-z\| .
\end{aligned}
$$

According to the upper bound on the Lipschitz condition $L$, established in Theorem 2.2, we have

$$
\beta=1-\frac{2 L e^{\lambda a} a^{\alpha}}{\Gamma(\alpha+1)}>0
$$

and therefore, we conclude that

$$
\|y-z\| \leq \frac{1}{\beta}\left|y_{a}-z_{a}\right|
$$

and the theorem is proved.

THEOREm 3.2. Let $y$ and $z$ be the unique solutions of problems (1.1)(1.2) and (3.4)-(3.5), respectively, where in the later we assume that $\delta$ is such that $0<\alpha-\delta<1$. Then

$$
\|y-z\|=\mathcal{O}(\delta) .
$$

P r o o f. Taking (3.1) and (3.11) into account, for any $t \in[0, a]$, we have 
WELL-POSEDNESS AND NUMERICAL APPROXIMATION . . 1247

$$
\begin{aligned}
|y(t)-z(t)| & =\mid-\frac{e^{-\lambda t}}{\Gamma(\alpha)} \int_{0}^{a} e^{\lambda s}(a-s)^{\alpha-1} f(s, y(s)) d s \\
& +\frac{e^{-\lambda t}}{\Gamma(\alpha-\delta)} \int_{0}^{a} e^{\lambda s}(a-s)^{\alpha-\delta-1} f(s, z(s)) d s \\
& +\frac{e^{-\lambda t}}{\Gamma(\alpha)} \int_{0}^{t} e^{\lambda s}(t-s)^{\alpha-1} f(s, y(s)) d s \\
& -\frac{e^{-\lambda t}}{\Gamma(\alpha-\delta)} \int_{0}^{t} e^{\lambda s}(t-s)^{\alpha-\delta-1} f(s, z(s)) d s \mid .
\end{aligned}
$$

Since $e^{-\lambda t} \leq 1$, for all $t \geq 0$ and $\lambda>0$, and $e^{\lambda s} \leq e^{\lambda a}$, for all $0 \leq s$, then

$$
\begin{aligned}
|y(t)-z(t)| \leq & e^{\lambda a} \\
& \times\left(\int_{0}^{a}\left|\frac{(a-s)^{\alpha-1}}{\Gamma(\alpha)} f(s, y(s))-\frac{(a-s)^{\alpha-\delta-1}}{\Gamma(\alpha-\delta)} f(s, z(s))\right| d s\right. \\
& \left.+\int_{0}^{t}\left|\frac{(t-s)^{\alpha-1}}{\Gamma(\alpha)} f(s, y(s))-\frac{(t-s)^{\alpha-\delta-1}}{\Gamma(\alpha-\delta)} f(s, z(s))\right| d s\right) .
\end{aligned}
$$

Let us look at the first integral on the right-hand side of (3.15) first:

$$
\begin{aligned}
I_{1} & =\int_{0}^{a}\left|\frac{(a-s)^{\alpha-1}}{\Gamma(\alpha)} f(s, y(s))-\frac{(a-s)^{\alpha-\delta-1}}{\Gamma(\alpha-\delta)} f(s, z(s))\right| d s \\
& =\int_{0}^{a} \mid \frac{(a-s)^{\alpha-1}}{\Gamma(\alpha)} f(s, y(s))-\frac{(a-s)^{\alpha-1}}{\Gamma(\alpha)} f(s, z(s)) \\
& +\frac{(a-s)^{\alpha-1}}{\Gamma(\alpha)} f(s, z(s))-\frac{(a-s)^{\alpha-\delta-1}}{\Gamma(\alpha-\delta)} f(s, z(s)) \mid d s \\
& \leq \frac{L a^{\alpha}}{\Gamma(\alpha+1)}\|y-z\| \\
& +\int_{0}^{a}\left|\frac{(a-s)^{\alpha-1}}{\Gamma(\alpha)}-\frac{(a-s)^{\alpha-\delta-1}}{\Gamma(\alpha-\delta)}\right||f(s, z(s))| d s .
\end{aligned}
$$

Considering the function $G(x)=\frac{(a-s)^{x}}{\Gamma(x+1)}$, using the mean value theorem, we easily conclude that $\left|\frac{(a-s)^{\alpha-1}}{\Gamma(\alpha)}-\frac{(a-s)^{\alpha-\delta-1}}{\Gamma(\alpha-\delta)}\right| \leq C \delta$, where $C=\max _{x \in[\alpha-\delta-1, \alpha-\delta]}\left|G^{\prime}(x)\right|$, and therefore

$$
I_{1} \leq \frac{L a^{\alpha}}{\Gamma(\alpha+1)}\|y-z\|+C a\|f\| \delta .
$$


Proceeding similarly with the second integral on the right-hand side of (3.15), we could conclude that

$$
\begin{aligned}
I_{2} & =\int_{0}^{t}\left|\frac{(t-s)^{\alpha-1}}{\Gamma(\alpha)} f(s, y(s))-\frac{(t-s)^{\alpha-\delta-1}}{\Gamma(\alpha-\delta)} f(s, z(s))\right| d s \\
& \leq \frac{L t^{\alpha}}{\Gamma(\alpha+1)}\|y-z\|+C t\|f\| \delta \leq \frac{L a^{\alpha}}{\Gamma(\alpha+1)}\|y-z\|+C a\|f\| \delta,
\end{aligned}
$$

and therefore

$$
\|y-z\| \leq \frac{2 L a^{\alpha} e^{\lambda a}}{\Gamma(\alpha+1)}\|y-z\|+2 C a\|f\| \delta
$$

or

$$
\|y-z\| \leq \frac{2 C a\|f\| \delta}{\beta}
$$

where $\beta$ is given by (3.14). This completes the proof of the theorem.

TheOREm 3.3. Let $y$ and $z$ be the unique solutions of problems (1.1)(1.2) and (3.6)-(3.7), respectively. Then

$$
\|y-z\|=\mathcal{O}(\delta)
$$

P r o o f. Taking (3.1) and (3.12) into account, for any $t \in[0, a]$, we have

$$
\begin{aligned}
|y(t)-z(t)| & \leq y_{a}\left|e^{-\lambda t}-e^{-(\lambda-\delta) t}\right| \\
& +\frac{1}{\Gamma(\alpha)} \int_{0}^{a}\left|e^{\lambda s} f(s, y(s))-e^{(\lambda-\delta) s} f(s, z(s))\right|(a-s)^{\alpha-1} d s \\
& +\frac{1}{\Gamma(\alpha)} \int_{0}^{t}\left|e^{\lambda s} f(s, y(s))-e^{(\lambda-\delta) s} f(s, z(s))\right|(t-s)^{\alpha-1} d s .
\end{aligned}
$$

By using the mean value theorem with the function $g(x)=e^{-x t}$, we conclude that

$$
\left|e^{-\lambda t}-e^{-(\lambda-\delta) t}\right| \leq C_{1} \delta
$$

where $C_{1}=\max _{x \in[\lambda-\delta, \lambda]}\left|g^{\prime}(x)\right|$. 
WELL-POSEDNESS AND NUMERICAL APPROXIMATION . . 1249

Concerning the first integral on the right-hand side of (3.16):

$$
\begin{aligned}
J_{1} & =\int_{0}^{a}\left|e^{\lambda s} f(s, y(s))-e^{(\lambda-\delta) s} f(s, z(s))\right|(a-s)^{\alpha-1} d s \\
& \leq \int_{0}^{a}\left|e^{\lambda s} f(s, y(s))-e^{\lambda s} f(s, z(s))\right|(a-s)^{\alpha-1} d s \\
& +\int_{0}^{a}\left|e^{\lambda s} f(s, z(s))-e^{(\lambda-\delta) s} f(s, z(s))\right|(a-s)^{\alpha-1} d s \\
& \leq \frac{e^{\lambda a} L a^{\alpha}}{\alpha}\|y-z\|+C_{2}\|f\| \delta,
\end{aligned}
$$

where by the mean value theorem, $C_{2}=\max _{x \in[\lambda-\delta, \lambda]}\left|h^{\prime}(x)\right|$, where $h(x)=$ $e^{x s}, s \in[0, a]$.

Proceeding analogously with the second integral in (3.16), we conclude that

$$
\begin{aligned}
J_{2} & =\frac{1}{\Gamma(\alpha)} \int_{0}^{t}\left|e^{\lambda s} f(s, y(s))-e^{(\lambda-\delta) s} f(s, z(s))\right|(t-s)^{\alpha-1} d s \\
& \leq \frac{e^{\lambda a} L a^{\alpha}}{\alpha}\|y-z\|+C_{2}\|f\| \delta .
\end{aligned}
$$

Hence

or

$$
\|y-z\| \leq C_{1} y_{a} \delta+\frac{2 e^{\lambda a} L a^{\alpha}}{\Gamma(\alpha+1)}\|y-z\|+2 C_{2}\|f\| \delta,
$$

$$
\|y-z\| \leq \frac{C_{1}+2 C_{2}\|f\|}{\beta} \delta
$$

with $\beta$ defined in (3.14). Thus, the theorem is proved.

ThEOREM 3.4. Let $y$ and $z$ be the unique solutions of problems (1.1)(1.2) and (3.8)-(3.9), respectively. Then

$$
\|y-z\|=\mathcal{O}(\|f-\tilde{f}\|)
$$

P r o o f. Taking (3.1) and (3.13) into account, for any $t \in[0, a]$, we have

$$
|y(t)-z(t)| \leq \frac{e^{\lambda a}}{\Gamma(\alpha)}\left(I_{1}+I_{2}\right),
$$

where $I_{1}=\int_{0}^{a}(a-s)^{\alpha-1}|f(s, y(s))-\tilde{f}(s, z(s))| d s$ and $I_{2}=\int_{0}^{t}(t-s)^{\alpha-1}|f(s, y(s))-\tilde{f}(s, z(s))| d s$. Since 
and, analogously

$$
\begin{aligned}
I_{1} & \leq \int_{0}^{a}(a-s)^{\alpha-1}|f(s, y(s))-f(s, z(s))| d s \\
& +\int_{0}^{a}(a-s)^{\alpha-1}|f(s, z(s))-\tilde{f}(s, z(s))| d s \\
& \leq \frac{L a^{\alpha}}{\alpha}\|y-z\|+\frac{a^{\alpha}}{\alpha}\|f-\tilde{f}\|,
\end{aligned}
$$

then

$$
\begin{aligned}
I_{2} & \leq \frac{L t^{\alpha}}{\alpha}\|y-z\|+\frac{t^{\alpha}}{\alpha}\|f-\tilde{f}\| \\
& \leq \frac{L a^{\alpha}}{\alpha}\|y-z\|+\frac{a^{\alpha}}{\alpha}\|f-\tilde{f}\|,
\end{aligned}
$$

$$
\|y-z\| \leq \frac{2 L a^{\alpha} e^{\lambda a}}{\Gamma(\alpha+1)}\|y-z\|+2 \frac{a^{\alpha} e^{\lambda a}}{\Gamma(\alpha+1)}\|f-\tilde{f}\|,
$$

and the result of the theorem follows.

\section{Numerical method}

From Lemma 2.1 we have an equivalence between tempered initial value problems and nonlinear Volterra integral equations. On the other hand, considering the initial value problem (2.1), with $\alpha \in(0,1)$, the solution of (2.1) is the solution of the integral equation:

$$
y(t)=c_{0} e^{-\lambda t}+\frac{1}{\Gamma(\alpha)} \int_{0}^{t} e^{-\lambda(t-s)}(t-s)^{\alpha-1} f(s, u(s)) d s, \quad 0<\alpha<1 .
$$

We can rewrite the integral equation (4.1) as

$$
u(t)=c_{0}+\frac{1}{\Gamma(\alpha)} \int_{0}^{t}(t-s)^{\alpha-1} g(s, u(s)) d s,
$$

where $c_{0}=y(0)$, and

$$
u(s)=e^{\lambda s} y(s) \text { and } g(s, u(s))=e^{\lambda s} f\left(s, e^{-\lambda s} u(s)\right) .
$$

Then, $u$ is a solution of the (non-tempered) Caputo-type initial value problem

$$
\begin{aligned}
& { }_{0} \mathcal{D}_{t}^{C, \alpha}(u(t))=g(t, u(t)), \quad t>0, \\
& u(0)=c_{0} .
\end{aligned}
$$

Therefore taking the equivalence between initial value tempered fractional equations and Caputo-type initial value problems into account, from Theorem 6, 2] we obtain the following result for tempered fractional equations of order $\alpha$, with $\alpha \in(0,1)$. 
WELL-POSEDNESS AND NUMERICAL APPROXIMATION ... 1251

Theorem 4.1. Let $\alpha \in(0,1)$ and assume that $f:[0, b] \times[c, d] \rightarrow \mathbb{R}$ is continuous and satisfies a Lipschitz condition with respect to the second variable.

If $y_{1}$ and $y_{2}$ are are two solutions of the tempered differential equations

$$
\mathbb{D}^{\alpha, \lambda}\left(y_{j}(t)\right)=f\left(t, y_{j}(t)\right), \quad j=1,2,
$$

subject to the initial conditions $y_{j}(0)=y_{j 0}, j=1,2$, respectively, where $y_{10} \neq y_{20}$.

Then for all $t \in[0, b]$ we have $y_{1}(t) \neq y_{2}(t)$.

From Theorem 4.1 we can conclude that a solution of a tempered fractional differential equation of order $\alpha \in(0,1)$ is uniquely defined by a condition that can be specified at any point $t \in[0, b]([0, b]$ is the interval where the solution of the fractional problem exist).

On the other hand, Theorem 4.1 will be crucial to properly define the ideas of the numerical methods that we present next.

From Theorem 4.1 it follows that for the solution of (1.1) that passes through the point $\left(a, \exp (-\lambda a) y_{a}\right)$, we are able to find at most one point $\left(0, y_{0}\right)$ that also lies on the same solution trajectory. In order to obtain an approximation of $y(0)$ we propose a shooting algorithm based on the bisection method. Let $y_{1}$ and $y_{2}$ be the solutions of (4.6) with initial values $y_{01}$ and $y_{02}$, such that $y_{1}(a)<\exp (-\lambda a) y_{a}<y_{2}(a)$, the bisection method provide successive approximations for $y(0)$ until the distance between the two last approximations does not exceed a given tolerance $\epsilon$.

In our numerical experiments, for illustration purposes, we have used the bisection method to find the initial value, but obviously, other methods can be used as easily as this, as the secant method, for instance.

To evaluate the value of $y(a)$ we need a numerical method to solve the initial value problems

$$
\begin{aligned}
& \mathbb{D}^{\alpha, \lambda}(y(t))=f(t, y(t)), \quad t \in(0, a], \\
& y(0)=y_{0} .
\end{aligned}
$$

This will be straightforward if we take relationship (1.3) into account. In fact, defining the functions $u$ and $g$ as in (4.3), we can use any available solver for (non-tempered) Caputo-type initial value problems to determine the solution $u$ of

$$
\begin{aligned}
& { }_{0} \mathcal{D}_{t}^{C, \alpha}(u(t))=g(t, u(t)), \quad t \in(0, a], \\
& u(0)=y_{0},
\end{aligned}
$$

and then the solution of (4.7)-(4.8) will be given by $y(t)=e^{-\lambda t} u(t)$. 


\section{Numerical results}

In this section we present some numerical examples to illustrate the efficiency of the numerical algorithm.

5.1. Approximating the solution of the terminal value problem (1.1)-(1.2). In this subsection we present 3 examples and the method that we apply for each one, depends on the nature of the differential equation and regularity of the solution.

The first example is a linear fractional differential equation with a smooth solution.

EXAMPLE 5.1.

$$
\begin{aligned}
& \mathbb{D}^{\alpha, \lambda}(y(t))=e^{-\lambda t}\left(\frac{3 \Gamma(3) t^{2-\alpha}}{4 \Gamma(3-\alpha)}+\frac{\Gamma(5) t^{4-\alpha}}{\Gamma(5-\alpha)}+c_{\alpha, \lambda}\left(t^{4}+\frac{3 t^{2}}{4}\right)\right) \\
& \quad-c_{\alpha, \lambda} y(t), \quad t>0, \\
& y(0.5)=\frac{e^{-\lambda / 2}}{4},
\end{aligned}
$$

where $\quad c_{\alpha, \lambda}=\frac{\Gamma(\alpha+1)}{2^{1-\alpha} e^{\lambda / 2}}$ and whose analytical solution is given by

$y(t)=\left(t^{4}+\frac{3 t^{2}}{4}\right) e^{-\lambda t}$.

The second example is a nonlinear fractional differential equation with a smooth solution defined by:

EXAMPLE 5.2.

$$
\begin{aligned}
& \mathbb{D}^{\alpha, \lambda}(y(t))=e^{-\lambda t}\left(\frac{\Gamma(3) t^{2-\alpha}}{\Gamma(3-\alpha)} t^{2-\alpha}-3 t^{4} \exp (-\lambda t)\right)+3 y^{2}, \quad t>0, \\
& y(0.5)=\frac{e^{-\lambda / 2}}{4}
\end{aligned}
$$

whose analytical solution is given by $y(t)=e^{-\lambda t} t^{2}$.

The third example is a linear fractional differential equation with a solution whose second derivative has a singularity at $t=0$.

EXAMPLE 5.3.

$$
\begin{aligned}
& \mathbb{D}^{\alpha, \lambda}(y(t))=e^{-\lambda t} \frac{\Gamma(5 / 2) t^{2-\alpha}}{\Gamma(5 / 2-\alpha)} t^{3 / 2-\alpha}, \quad t>0, \\
& y(0.5)=e^{-\lambda / 2} \sqrt{\frac{1}{2^{3}}}
\end{aligned}
$$

whose analytical solution is given by $y(t)=e^{-\lambda t} t^{3 / 2}$. 
WELL-POSEDNESS AND NUMERICAL APPROXIMATION ... 1253

In what follows we consider Examples 5.1, 5.2 and 5.3 with several values of $\alpha$ and $\lambda=2$.

In order to compute $y(0)$ the bisection method was used with $\epsilon=10^{-10}$ and the approximate solution of each one of IVP was computed with the three methods listed bellow.

- Method 1. Fractional backward difference based on quadrature (see, for example, [4]).

- Method 2. This numerical method can be seen as a generalization of the classical one-step Adams-Bashforth-Moulton scheme for firstorder equations (cf. [5]) and is appropriate to obtain a numerical solution of the non-linear problems.

- Method 3. In this method we consider an integral formulation of the initial value problem (4.9)-(4.10) and a nonpolynomial approximation of the solution (cf. [11]). This method is appropriate to approximate the solution of problems whose solution is not smooth.

We denote the absolute errors by $e_{\epsilon}^{h}(t)=\left|y(t)-y_{\epsilon}^{h}(t)\right|$, where $y_{\epsilon}^{h}$ is an approximate solution of $y$ using the algorithm with stepsize $h=\frac{a}{N}$ and the value $y_{0} \sim y(0)$ was obtained by the bisection method with tolerance $\epsilon$.

The absolute errors for $t=a=0.5$ and $t=1$ and the obtained values of $y(0)$ for the Examples [5.1, 5.2 and 5.3 are presented in Tables 1, 3] and 5, respectively.

In Tables 2, 4 and 6 the maximum of the absolute errors, $\left\|e^{a / N}\right\|_{\infty}=\max _{0 \leq i \leq N} e_{\epsilon}^{a / N}\left(t_{i}\right)$, and the experimental orders of convergence, $p_{h}=\frac{\log \left(\left\|e^{h}\right\|_{\infty} /\left\|e^{h / 2}\right\|_{\infty}\right)}{\log (2)}$, are listed.

In Table 11 we observe that the absolute error for the point where the boundary condition is imposed, does not decrease as the step-size goes smaller, although we are comparing very small quantities. On the other hand, for the approximate solution of Example 5.2 the absolute error at the boundary point decreases as the step-size $h$ decreases (cf. Table 3) and decreases with convergence order $1+\alpha$. As shown in Figure 1 a good agreement was obtained between the numerical and the analytical solutions.

In Tables 2 and 4 the experimental orders of convergence are listed, and we observe that the corresponding to Examples 5.1 and 5.2 are approximately $2-\alpha$ and $1+\alpha$, respectively. The results are in agreement with the theoretical result proved in [4, for Method 1, and with the conjecture of Diethelm et al. [5], for Method 2. 


\begin{tabular}{|l|ccc|ccc|ccc|}
\hline \multirow{3}{*}{$h$} & \multicolumn{3}{|c|}{$\alpha=1 / 4$} & \multicolumn{3}{c|}{$\alpha=1 / 2$} & \multicolumn{3}{c|}{$\alpha=2 / 3$} \\
\cline { 2 - 10 } & $y(0)$ & $e_{\epsilon}^{h}(0.5)$ & $e_{\epsilon}^{h}(1)$ & $y(0)$ & $e_{\epsilon}^{h}(0.5)$ & $e_{\epsilon}^{h}(1)$ & $y(0)$ & $e_{\epsilon}^{h}(0.5)$ & $e_{\epsilon}^{h}(1)$ \\
\hline $1 / 10$ & -0.001564 & $2.275 \times 10^{-11}$ & $6.382 \times 10^{-4}$ & -0.005128 & $1.975 \times 10^{-11}$ & $2.297 \times 10^{-3}$ & -0.009602 & $1.279 \times 10^{-11}$ & $4.604 \times 10^{-3}$ \\
$1 / 20$ & -0.000510 & $2.938 \times 10^{-11}$ & $2.045 \times 10^{-4}$ & -0.001906 & $2.844 \times 10^{-11}$ & $8.438 \times 10^{-4}$ & -0.003922 & $2.719 \times 10^{-11}$ & $1.866 \times 10^{-3}$ \\
$1 / 40$ & -0.000163 & $9.517 \times 10^{-12}$ & $6.446 \times 10^{-5}$ & -0.000698 & $1.745 \times 10^{-11}$ & $3.063 \times 10^{-4}$ & -0.001587 & $2.411 \times 10^{-11}$ & $7.510 \times 10^{-4}$ \\
$1 / 80$ & -0.000051 & $3.158 \times 10^{-11}$ & $2.006 \times 10^{-5}$ & -0.000253 & $3.025 \times 10^{-11}$ & $1.103 \times 10^{-4}$ & -0.000638 & $3.731 \times 10^{-11}$ & $3.007 \times 10^{-4}$ \\
$1 / 160$ & -0.000016 & $4.922 \times 10^{-12}$ & $6.189 \times 10^{-6}$ & -0.000032 & $8.683 \times 10^{-11}$ & $3.948 \times 10^{-4}$ & -0.000255 & $2.321 \times 10^{-11}$ & $1.200 \times 10^{-4}$ \\
$1 / 320$ & -0.000005 & $4.251 \times 10^{-12}$ & $1.896 \times 10^{-6}$ & -0.000091 & $1.230 \times 10^{-11}$ & $1.408 \times 10^{-4}$ & -0.000102 & $1.198 \times 10^{-11}$ & $4.781 \times 10^{-5}$ \\
\hline
\end{tabular}

TABLE 1. Example 5.1 with several values of $\alpha$. Comparison with the exact solution at $t=0.5$ (the value that defines the boundary condition) and $t=1$ with several values of the stepsize $h$.

\begin{tabular}{|l|cc|cc|cc|}
\hline \multirow{3}{*}{$h$} & \multicolumn{2}{|c|}{$\alpha=1 / 4$} & \multicolumn{2}{c|}{$\alpha=1 / 2$} & \multicolumn{2}{c|}{$\alpha=2 / 3$} \\
\cline { 2 - 7 } & $\left\|e^{h}\right\|_{\infty}$ & $p_{h}$ & $\left\|e^{h}\right\|_{\infty}$ & $p_{h}$ & $\left\|e^{h}\right\|_{\infty}$ & $p_{h}$ \\
\hline $1 / 10$ & $1.564 \times 10^{-3}$ & - & $5.128 \times 10^{-3}$ & - & $9.602 \times 10^{-3}$ & - \\
$1 / 20$ & $5.098 \times 10^{-4}$ & 1.62 & $1.906 \times 10^{-3}$ & 1.43 & $3.922 \times 10^{-3}$ & 1.29 \\
$1 / 40$ & $1.626 \times 10^{-4}$ & 1.65 & $6.978 \times 10^{-4}$ & 1.45 & $1.587 \times 10^{-3}$ & 1.31 \\
$1 / 80$ & $5.107 \times 10^{-5}$ & 1.67 & $2.527 \times 10^{-4}$ & 1.47 & $6.381 \times 10^{-4}$ & 1.31 \\
$1 / 160$ & $1.586 \times 10^{-5}$ & 1.69 & $9.084 \times 10^{-5}$ & 1.48 & $2.553 \times 10^{-4}$ & 1.32 \\
$1 / 320$ & $4.883 \times 10^{-6}$ & 1.70 & $3.249 \times 10^{-5}$ & 1.48 & $1.019 \times 10^{-4}$ & 1.33 \\
\hline
\end{tabular}

TABLE 2. Example 5.1 with several values of $\alpha$. Maximum of absolute errors and experimental orders of convergence.

\begin{tabular}{|l|ccc|ccc|ccc|}
\hline & \multicolumn{3}{|c|}{$\alpha=1 / 4$} & & \multicolumn{3}{c|}{$\alpha=1 / 2$} & \multicolumn{3}{c|}{$\alpha=2 / 3$} \\
\cline { 2 - 10 }$h$ & $y(0)$ & $e_{\epsilon}^{h}(0.5)$ & $e_{\epsilon}^{h}(1)$ & $y(0)$ & $e_{\epsilon}^{h}(0.5)$ & $e_{\epsilon}^{n}(1)$ & $y(0)$ & $e_{\epsilon}^{h}(0.5)$ & $e_{\epsilon}^{h}(1)$ \\
\hline $1 / 20$ & $9.313 \times 10^{-11}$ & $3.014 \times 10^{-3}$ & $5.280 \times 10^{-3}$ & $9.313 \times 10^{-11}$ & $8.484 \times 10^{-4}$ & $1.487 \times 10^{-3}$ & $9.313 \times 10^{-11}$ & $3.440 \times 10^{-4}$ & $6.534 \times 10^{-4}$ \\
$1 / 40$ & $9.313 \times 10^{-11}$ & $1.364 \times 10^{-3}$ & $2.386 \times 10^{-3}$ & $9.313 \times 10^{-11}$ & $3.273 \times 10^{-4}$ & $5.559 \times 10^{-4}$ & $9.313 \times 10^{-11}$ & $1.214 \times 10^{-4}$ & $2.181 \times 10^{-4}$ \\
$1 / 80$ & $9.313 \times 10^{-11}$ & $5.908 \times 10^{-4}$ & $1.038 \times 10^{-3}$ & $9.313 \times 10^{-11}$ & $1.214 \times 10^{-4}$ & $2.028 \times 10^{-4}$ & $9.313 \times 10^{-11}$ & $4.121 \times 10^{-5}$ & $7.127 \times 10^{-5}$ \\
$1 / 160$ & $9.313 \times 10^{-11}$ & $2.504 \times 10^{-4}$ & $4.427 \times 10^{-4}$ & $9.313 \times 10^{-11}$ & $4.412 \times 10^{-5}$ & $7.302 \times 10^{-5}$ & $9.313 \times 10^{-11}$ & $1.369 \times 10^{-5}$ & $2.302 \times 10^{-5}$ \\
$1 / 320$ & $9.313 \times 10^{-11}$ & $1.051 \times 10^{-4}$ & $1.872 \times 10^{-4}$ & $9.313 \times 10^{-11}$ & $1.587 \times 10^{-5}$ & $2.611 \times 10^{-5}$ & $9.313 \times 10^{-11}$ & $4.486 \times 10^{-6}$ & $7.382 \times 10^{-6}$ \\
\hline
\end{tabular}

TABLE 3. Example 5.2 with several values of $\alpha$. Comparison with the exact solution at $t=0.5$ (the value in the boundary condition) and $t=1$ with several values of the stepsize $h$ (shooting method with Method 2 to solve the IVP).

In Figures 2 the absolute errors of the approximate solutions of Examples 5.1 and 5.2, with several values of $\alpha$, are plotted for stepsize $h=1 / 160$. For Example 5.1 we observe that the absolute error is minimum at the point $t=a$ and for Example 5.2 the absolute error is minimum at the point $t=0$. For both examples the absolute error decreases with the value of $\alpha$. 
WELL-POSEDNESS AND NUMERICAL APPROXIMATION . . 1255

\begin{tabular}{|l|cc|cc|cc|}
\hline \multirow{3}{*}{$h$} & \multicolumn{2}{|c|}{$\alpha=1 / 4$} & \multicolumn{2}{c|}{$\alpha=1 / 2$} & \multicolumn{2}{c|}{$\alpha=2 / 3$} \\
\cline { 2 - 7 } & $\left\|e^{h}\right\|_{\infty}$ & $p_{h}$ & $\left\|e^{h}\right\|_{\infty}$ & $p_{h}$ & $\left\|e^{h}\right\|_{\infty}$ & $p_{h}$ \\
\hline $1 / 20$ & $5.306 \times 10^{-3}$ & - & $1.494 \times 10^{-3}$ & - & $6.565 \times 10^{-4}$ & - \\
$1 / 40$ & $2.399 \times 10^{-3}$ & 1.15 & $5.592 \times 10^{-4}$ & 1.42 & $2.194 \times 10^{-4}$ & 1.58 \\
$1 / 80$ & $1.043 \times 10^{-3}$ & 1.20 & $2.041 \times 10^{-4}$ & 1.45 & $7.182 \times 10^{-5}$ & 1.61 \\
$1 / 160$ & $4.450 \times 10^{-4}$ & 1.23 & $7.352 \times 10^{-5}$ & 1.47 & $2.322 \times 10^{-5}$ & 1.63 \\
$1 / 320$ & $1.881 \times 10^{-4}$ & 1.24 & $2.630 \times 10^{-5}$ & 1.48 & $7.452 \times 10^{-6}$ & 1.64 \\
\hline
\end{tabular}

TABLE 4. Example 5.2 with several values of $\alpha$ (shooting method with Method 2 to solve the IVP).
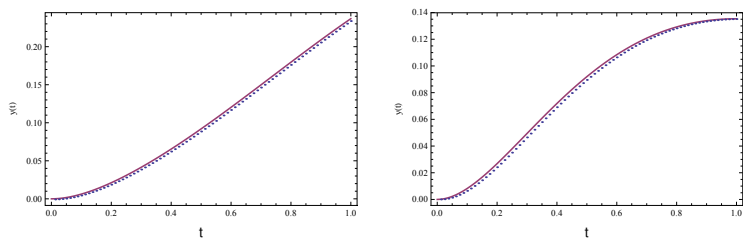

Figure 1. Comparison between exact solution (solid line) and approximate solution (dashed line) obtained with stepsize $h=0.025$. Left: Example 5.1 with $\alpha=1 / 2$. Right: Example 5.2 with $\alpha=1 / 2$.
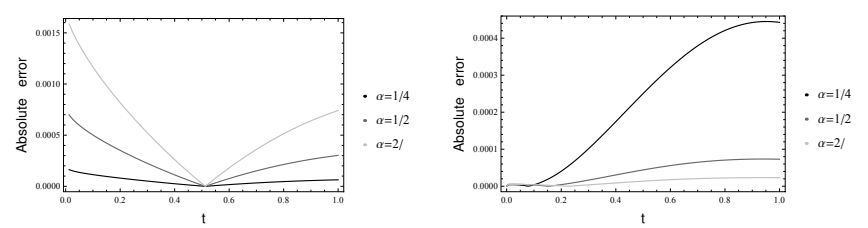

Figure 2. Plot of error function $\left|y(t)-y_{\epsilon}^{h}(t)\right|$, with $h=$ 1/160, for Example 5.1 (left) and for Example [5.2 (right), with $\alpha=1 / 4, \alpha=1 / 2$ and $\alpha=2 / 3$.

In Tables 5 and 6 we compare the results obtained with the shooting method and $y_{0}$ given by Method 1 and Method 3 on the space $V_{h, 1}^{1 / 2}$. We observe that the error at $t=0$ is smaller when $y_{0}$ is obtained by Method 3. However, both methods converge to zero with convergence order 1.5, approximately. 
In Figure 3 we can observe a good agreement between the numerical solution obtained with the shooting method and $y_{0}$ given by Method 1 and Method 3 on the space $V_{h, 1}^{1 / 2}$ and the analytical solution.

From Figure 4, right, we observe that the absolute error of the approximate solution, $y^{h}$, is very small, namely, the maximum of the absolute error is approximately $6 \times 10^{-11}$, even with a stepsize not too small, $h=1 / 20$. This is not surprising, once the solution belongs to $V_{2}^{1 / 2}=\left\langle 1, t^{1 / 2}, t, t^{3 / 2}\right\rangle$.

\begin{tabular}{|l|ccc|ccc|}
\hline & \multicolumn{3}{|c|}{ Method 1 } & \multicolumn{3}{c|}{ Method 3 } \\
\cline { 2 - 7 }$h$ & $y(0)$ & $e_{\epsilon}^{h}(0.5)$ & $e_{\epsilon}^{h}(1)$ & $y(0)$ & $e_{\epsilon}^{h}(0.5)$ & $e_{\epsilon}^{h}(1)$ \\
\hline $1 / 20$ & $3.037 \times 10^{-3}$ & $1.340 \times 10^{-12}$ & $1.814 \times 10^{-5}$ & $5.821 \times 10^{-11}$ & $7.844 \times 10^{-5}$ & $6.302 \times 10^{-6}$ \\
$1 / 40$ & $1.121 \times 10^{-3}$ & $8.904 \times 10^{-12}$ & $4.458 \times 10^{-6}$ & $5.821 \times 10^{-11}$ & $2.955 \times 10^{-5}$ & $1.657 \times 10^{-6}$ \\
$1 / 80$ & $4.081 \times 10^{-4}$ & $1.305 \times 10^{-11}$ & $1.105 \times 10^{-6}$ & $5.821 \times 10^{-11}$ & $1.098 \times 10^{-5}$ & $4.286 \times 10^{-7}$ \\
$1 / 160$ & $1.472 \times 10^{-4}$ & $1.531 \times 10^{-11}$ & $2.750 \times 10^{-7}$ & $5.821 \times 10^{-11}$ & $4.119 \times 10^{-7}$ & $1.096 \times 10^{-7}$ \\
$1 / 320$ & $5.275 \times 10^{-5}$ & $6.367 \times 10^{-12}$ & $6.859 \times 10^{-8}$ & $5.821 \times 10^{-11}$ & $1.054 \times 10^{-7}$ & $2.785 \times 10^{-8}$ \\
\hline
\end{tabular}

TABLE 5. Example 5.3 with $\alpha=1 / 2$. Comparison with the exact solution at $t=0, t=0.5$ (the value that defines the boundary condition) and $t=1$ with several values of the stepsize $h$ (shooting method with Method 1 and Method 3 on the space $V_{h 1}^{1 / 2}\left(c_{1}=1 / 3, c_{2}=1\right)$ to solve the IVP).

\begin{tabular}{|l|cc|cc|}
\hline \multirow{2}{*}{$h$} & \multicolumn{2}{|c|}{ Method 1 } & \multicolumn{2}{c|}{ Method 3 } \\
\cline { 2 - 5 } & $\left\|e^{h}\right\|_{\infty}$ & $p_{h}$ & $\left\|e^{h}\right\|_{\infty}$ & $p_{h}$ \\
\hline $1 / 20$ & $3.304 \times 10^{-3}$ & - & $7.844 \times 10^{-5}$ & - \\
$1 / 40$ & $1.121 \times 10^{-3}$ & 1.44 & $2.955 \times 10^{-5}$ & 1.41 \\
$1 / 80$ & $4.081 \times 10^{-4}$ & 1.46 & $1.098 \times 10^{-5}$ & 1.43 \\
$1 / 160$ & $1.472 \times 10^{-4}$ & 1.47 & $3.981 \times 10^{-6}$ & 1.46 \\
$1 / 320$ & $5.275 \times 10^{-5}$ & 1.48 & $1.425 \times 10^{-6}$ & 1.48 \\
\hline
\end{tabular}

TABLE 6. Example 5.3 with $\alpha=1 / 2$. Maximum of absolute errors and experimental orders of convergence (shooting method with Method 1 and Method 3 on the space $V_{h 1}^{1 / 2}$ $\left(c_{1}=1 / 3, c_{2}=1\right)$. 
WELL-POSEDNESS AND NUMERICAL APPROXIMATION ... 1257

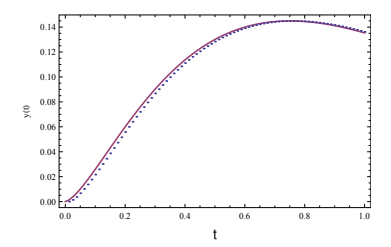

Figure 3. Comparison between the exact solution (solid line) of Example 5.3 and approximate solution (dashed line) obtained by shooting method with Method 3 on the space $V_{h 1}^{1 / 2}(h=0.025)$.
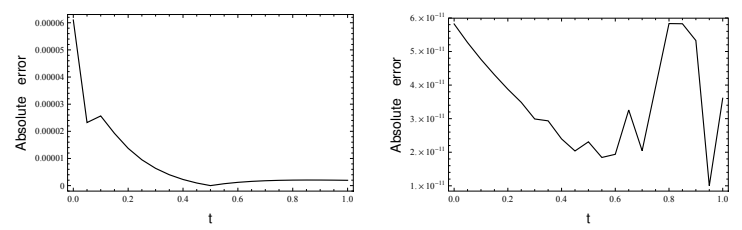

FiguRe 4. Plot of error function $\left|y(t)-y^{h}(t)\right|$, with $h=1 / 20$, from Example 5.3. Left: Shooting method with Method 3 on the space $V_{h 1}^{1 / 2}$. Right: Shooting method with Method 3 on the space $V_{h 2}^{1 / 2}$.

5.2. Dependence on the problem parameters. In this subsection we consider a nonlinear problem and illustrate numerically the stability of the problem.

Let us consider the tempered fractional differential equation

$$
\begin{aligned}
& \mathbb{D}^{\alpha, \lambda}(y(t))=2 t+\frac{\Gamma(\alpha+1)}{3 \exp (\lambda a) a^{\alpha}} \sin (u)=f(t, u), \quad t>0, \\
& y(a)=y_{a}
\end{aligned}
$$

with $\lambda=2, a=1 / 2, y_{a}=1$ and $\alpha=1 / 2$. Note that the function $f$ satisfies the assumptions of Theorems 3.1]3.4. In this case the exact solution of Example 5.1 is unknown. 
Let us consider the perturbed problems

$$
\begin{aligned}
& \mathbb{D}^{\alpha, \lambda}(z(t))=f(t, z), \quad t>0, \\
& z(a)=y_{a}+\epsilon_{b c}, \\
& \mathbb{D}^{\alpha, \lambda}(z(t))=f(t, z)+\epsilon_{f}, \quad t>0, \\
& z(a)=y_{a}, \\
& \mathbb{D}^{\alpha, \lambda+\epsilon_{\lambda}}(z(t))=f(t, z), \quad t>0, \\
& z(a)=y_{a}, \\
& \mathbb{D}^{\alpha+\epsilon_{\alpha}, \lambda}(z(t))=f(t, z), \quad t>0, \\
& z(a)=y_{a} .
\end{aligned}
$$

The obtained $\max _{1 \leq i \leq N}\left\|y_{i}-z_{i}\right\|=\|y-z\|_{\infty}$ are presented in Tables 17, 8 and 9, where $y_{i}$ and $z_{i}$ are the obtained numerical approximations of $y(t)$ and $z(t)$ at the discretization points $t=t_{i}=i h$, with $h=a / N$, and $z$ is the solution of the perturbed problems (5.2), (5.3) and (5.4), respectively.

In Table 7 we present the results obtained when we compare the problems (5.1) and (5.2), when the boundary condition suffers a perturbation.

In Table 8 we present the results obtained when we compare the problems (5.1) and (5.3), when the source function $f$ has a perturbation, $\epsilon_{f}$.

Finally, in Table 9 we illustrate how the solution of (5.4) varies with $\epsilon_{\lambda}$.

According to the numerical results in Tables 7, 8] and 9, we see that, independently of the used step size $h$, we have $\|y-z\|_{\infty} \sim \epsilon_{b c},\|y-z\|_{\infty} \sim \epsilon_{f}$ and $\|y-z\|_{\infty} \sim \epsilon_{\lambda}$, if $z$ is the approximate solution of the problems (5.2), (5.3) and (5.4), respectively. The numerical results are in agreement with the theoretical results proved in Theorems 3.1 , 3.3 and 3.4 .

\begin{tabular}{|l|ccccc|}
\hline & \multicolumn{5}{|c|}{ Values of $\epsilon_{b c}$} \\
\cline { 2 - 6 }$h$ & 0.1 & 0.01 & 0.001 & 0.0001 & 0.00001 \\
\hline $1 / 20$ & $2.5704 \times 10^{-1}$ & $2.5518 \times 10^{-2}$ & $2.5499 \times 10^{-3}$ & $2.5498 \times 10^{-4}$ & $2.5497 \times 10^{-5}$ \\
$1 / 40$ & $2.5712 \times 10^{-1}$ & $2.5525 \times 10^{-2}$ & $2.5507 \times 10^{-3}$ & $2.5506 \times 10^{-4}$ & $2.5505 \times 10^{-5}$ \\
$1 / 80$ & $2.5715 \times 10^{-1}$ & $2.5528 \times 10^{-2}$ & $2.5510 \times 10^{-3}$ & $2.5508 \times 10^{-4}$ & $2.5508 \times 10^{-5}$ \\
$1 / 160$ & $2.5716 \times 10^{-1}$ & $2.5529 \times 10^{-2}$ & $2.5519 \times 10^{-3}$ & $2.5509 \times 10^{-4}$ & $2.5509 \times 10^{-5}$ \\
\hline
\end{tabular}

TABLE 7. Maximum of the absolute errors, $\left|y^{h}-z^{h}\right|$, where $y^{h}$ is the numerical solution of problem (5.1) and $z^{h}$ the numerical solution of the problem (5.2) with several values of $\epsilon_{b c}$. 
WELL-POSEDNESS AND NUMERICAL APPROXIMATION ... 1259

\begin{tabular}{|l|ccccc|}
\hline & \multicolumn{5}{|c|}{ Values of $\epsilon_{f}$} \\
\cline { 2 - 6 }$h$ & 0.1 & 0.01 & 0.001 & 0.0001 & 0.00001 \\
\hline $1 / 20$ & $7.8815 \times 10^{-2}$ & $7.8841 \times 10^{-3}$ & $7.8844 \times 10^{-4}$ & $7.8844 \times 10^{-5}$ & $7.8845 \times 10^{-6}$ \\
$1 / 40$ & $7.8818 \times 10^{-2}$ & $7.8844 \times 10^{-3}$ & $7.8847 \times 10^{-4}$ & $7.8847 \times 10^{-5}$ & $7.8847 \times 10^{-6}$ \\
$1 / 80$ & $7.8819 \times 10^{-2}$ & $7.8845 \times 10^{-3}$ & $7.8848 \times 10^{-4}$ & $7.8848 \times 10^{-5}$ & $7.8848 \times 10^{-6}$ \\
$1 / 160$ & $7.8820 \times 10^{-2}$ & $7.8845 \times 10^{-3}$ & $7.8848 \times 10^{-4}$ & $7.8848 \times 10^{-5}$ & $7.8848 \times 10^{-6}$ \\
\hline
\end{tabular}

TABLE 8. Maximum of the absolute errors, $\left|y^{h}-z^{h}\right|$, where $y^{h}$ is the numerical solution of problem (5.1) and $z^{h}$ the numerical solution of the problem (5.3) with several values of $\epsilon_{f}$.

\begin{tabular}{|l|ccccc|}
\hline & \multicolumn{5}{|c|}{ Values of $\epsilon_{\lambda}$} \\
\cline { 2 - 6 }$h$ & 0.1 & 0.01 & 0.001 & 0.0001 & 0.00001 \\
\hline $1 / 20$ & $2.2715 \times 10^{-1}$ & $2.1483 \times 10^{-2}$ & $2.1364 \times 10^{-3}$ & $2.1352 \times 10^{-4}$ & $2.1351 \times 10^{-5}$ \\
$1 / 40$ & $2.2726 \times 10^{-1}$ & $2.1493 \times 10^{-2}$ & $2.1374 \times 10^{-3}$ & $2.1362 \times 10^{-4}$ & $2.1361 \times 10^{-5}$ \\
$1 / 80$ & $2.2729 \times 10^{-1}$ & $2.1496 \times 10^{-2}$ & $2.1377 \times 10^{-3}$ & $2.1365 \times 10^{-4}$ & $2.1364 \times 10^{-5}$ \\
$1 / 160$ & $2.2730 \times 10^{-1}$ & $2.1497 \times 10^{-2}$ & $2.1378 \times 10^{-3}$ & $2.1366 \times 10^{-4}$ & $2.1365 \times 10^{-5}$ \\
\hline
\end{tabular}

TABLE 9. Maximum of the absolute errors, $\left|y^{h}-z^{h}\right|$, where $y^{h}$ is the numerical solution of problem (5.1) and $z^{h}$ the numerical solution of the problem (5.4) with several values of $\epsilon_{\lambda}$.

In Figure [5] we present an approximate solution of the problem (5.4) with $\epsilon_{\alpha}=0.01$, and we observe that the variation is very small. We also plot the approximate solution of (5.1), for several values of $\alpha$, and we observe that the solution is an increasing function for $\alpha<0.5$ and a decreasing function for $\alpha \geq 0.5$. Finally, in Figure 6 we plot the absolute error $\mid y^{1 / 160}$ $z^{1 / 160} \mid$, where $y^{1 / 160}$ is the approximate solution of problem (5.1) and $z^{1 / 160}$ the approximate solution of the problem (5.4) with $\epsilon_{\lambda}=10^{-5}$. It can be observed that the absolute error is less than $\lambda \times 10^{-5}$ and the absolute error is maximum at the origin.

\section{Conclusions}

We have analyzed the well-posedness of ordinary tempered terminal value problems. Based on the relationship between non-tempered and tempered Caputo derivatives we have proposed three numerical schemes to approximate the solution of such problems. It should be noted that Method 3 has the advantage to properly deal with nonsmooth solutions which constitutes an important feature in the numerical approximation of fractional differential problems. In the future, we intend to extend it to partial and distributed differential problems. 

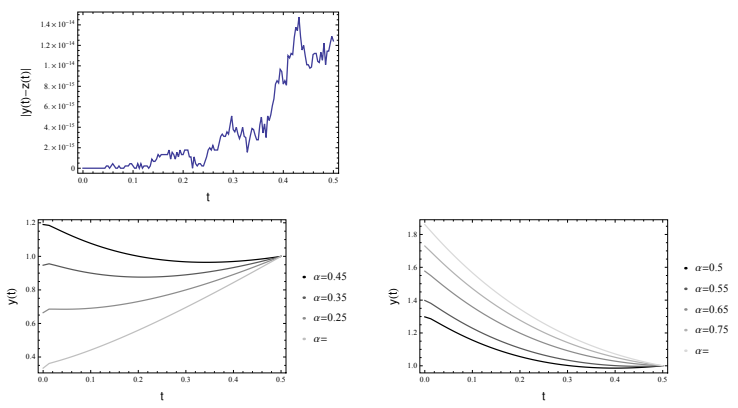

Figure 5. Top: Plot of the error function $|y(t)-z(t)|$, where $z$ is the approximate solution of (5.5) with $\epsilon_{\alpha}=0.01$. The approximate solutions are obtained using the Method 2 with $h=1 / 160$. Bottom: Approximate solutions of (5.5) with several values of $\alpha$.
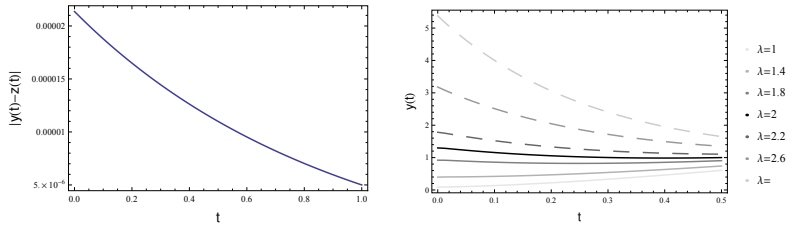

Figure 6. Left: Plot of the error function $|y(t)-z(t)|$, where $z$ is the approximate solution of the (5.4) with $\epsilon_{\alpha}=$ 0.00001 . The approximate solutions are obtained using the Method 2 with $h=1 / 160$. Right: Approximate solutions of (5.4) with several values of $\lambda$. The plots with dashed lines are related with the values of $\lambda$ greater than 2 .

\section{Acknowledgements}

The two authors acknowledge financial support from FCT "Fundação para a Ciência e a Tecnologia (Portuguese Foundation for Science and Technology)", through Project UID/MAT/00013/2013 and Project UID/MAT/ 00297/2013, respectively.

The authors wish to acknowledge the anonymous referees for their comments and suggestions which led to valuable improvements of the paper.

\section{References}

[1] B. Baeumer, M.M. Meerschaert, Tempered stable Lévy motion and transient super-diffusion. J. Comp. Appl. Mathem. 233 (2010), 24382448 . 
WELL-POSEDNESS AND NUMERICAL APPROXIMATION ... 1261

[2] N.D. Cong, H.T. Huan, Generation of nonlocal fractional dynamical systems by fractional differential equations. J. Integral Equations Applications, To appear.

[3] K. Diethelm, The Analysis of Fractional Differential Equations: An Application-Oriented Exposition Using Differential Operators of $\mathrm{Ca}$ puto Type. Springer (2010).

[4] K. Diethelm, An algorithm for the numerical solution of differential equations of fractional order. Electr. Trans. Numer. Anal. 5 (1997), $1-6$.

[5] K. Diethelm, N.J. Ford, A.D. Freed, Detailed error analysis for a fractional Adams method. Numerical Algorithms 36, No 1 (2004), 31-52.

[6] J.W. Deng, L.J. Zhao, Y.J. Wu, Fast predictor-corrector approach for the tempered fractional ordinary differential equations. Preprint arXiv:1502.00748 (2015).

[7] C. Li, W. Deng, L. Zhao, Well-posedness and numerical algorithm for the tempered fractional ordinary differential equations. Preprint arXiv:1501.00337v1 (2015).

[8] A. Liemert, A. Kienle, Fundamental solution of the tempered fractional diffusion equation. J. of Mathematical Physics 56 (2015), ID \# 113504.

[9] O. Marom, E. Momoniat, A comparison of numerical solutions of fractional diffusion models in finance. Nonl. Anal.: R.W.A. 10, No 6 (2009), 3435-3442.

[10] M.M. Meerschaert, Y. Zhang, B. Baeumer, Tempered anomalous diffusion in heterogeneous systems. Geophysical Research Letters 35 (2008), ID \# L17403.

[11] L. Morgado, M. Rebelo, N. Ford, Nonpolynomial collocation approximation of solutions to fractional differential equations, Fract. Calc. Appl. Anal. 16, No 4 (2013), 874-891; DOI: 10.2478/s13540-013-00543 ; https://www.degruyter.com/view/j/fca.2013.16.issue-4/

issue-files/fca.2013.16.issue-4.xml.

[12] F. Sabzikar, M.M. Meerschaert, J. Chen, Tempered fractional calculus. J. of Computational Physics 93, No 15 (2015), 14-28.

[13] L. Zhao, W. Deng, J.S. Hesthaven, Spectral Methods for Tempered Fractional Differential Equations. Preprint arXiv:1603.06511 (2016).

1 Centre of Mathematics, Pole CMAT-UTAD

and Department of Mathematics

University of Trás-os-Montes e Alto Douro, UTAD

Quinta de Prados 5001-801, Vila Real, PORTUGAL

e-mail:luisam@utad.pt

Received: May 4, 2017 
${ }^{2}$ Department of Mathematics and Centro de Matemática e Aplicações Universidade NOVA de Lisboa Quinta da Torre, 2829-516, Caparica, PORTUGAL

e-mail:msjr@fct.unl.pt

Revised: August 8, 2017

Please cite to this paper as published in:

Fract. Calc. Appl. Anal., Vol. 20, No 5 (2017), pp. 1239-1262,

DOI: $10.1515 /$ fca-2017-0065 